\title{
CD38 is highly expressed and affects the PI3K/Akt signaling pathway in cervical cancer
}

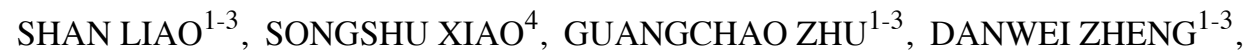 \\ JUNYU HE ${ }^{1-3}$, ZHEN PEI ${ }^{1-3}$, GUIYUAN LI ${ }^{1-3}$ and YANHONG ZHOU ${ }^{1-3}$

\begin{abstract}
${ }^{1}$ Hunan Provincial Tumor Hospital and The Tumor Hospital Affiliated to Xiangya School of Medicine, Central South University, Changsha, Hunan 410013; ${ }^{2}$ Key Laboratory of Carcinogenesis of Ministry of Health

Changsha, Hunan 410078; ${ }^{3}$ Hunan Key Laboratory of Nonresolving Inflammation and Cancer,

Disease Genome Research Center; ${ }^{4}$ Department of Gynecology and Obstetrics, The Third

Xiangya Hospital, Central South University, Changsha, Hunan 410013, P.R. China
\end{abstract} \\ and Key Laboratory of Carcinogenesis and Cancer Invasion of Ministry of Education, Cancer Research Institute,
}

Received July 18, 2014; Accepted September 2, 2014

DOI: $10.3892 / o r .2014 .3537$

\begin{abstract}
Cervical cancer is the second most common cancer and the fifth most deadly malignancy in females worldwide, affecting 500,000 individuals each year. It is the leading cause of cancer mortality among women in developing countries. Dysregulated activation of genes, such as CD44, SOX 9 and $S K P 2$, plays a role in cervical cancer. CD38 is known to be involved in activities typical of cell surface receptors, such as signaling for activation and proliferation events and heterotypic cell adhesion. CD38 contributes to disease progression and relapse in certain tumors, such as acute myeloid and chronic lymphocytic leukemia. To the best of our knowledge, there is currently no report on the relationship between CD38 and cervical cancer. Using qPCR, immunohistochemistry, and western blot analysis, the expression levels of CD38 were investigated and found to be upregulated in cervical cancer. CD38 was correlated with dysregulation of the phosphatidylinositol 3-kinase (PI3K)/Akt signaling pathway in cervical cancer tissues in vitro. At the same time, CD38 overexpression affected the expression of PI3K, Akt, MDM2 and p53 in vivo.
\end{abstract}

Correspondence to: Dr Yanhong Zhou or Professor Guiyuan Li, Key Laboratory of Carcinogenesis of Ministry of Health and Key Laboratory of Carcinogenesis and Cancer Invasion of Ministry of Education, Cancer Research Institute, Central South University, 110 Xiangya Road, Changsha, Hunan 410078, P.R. China

E-mail: zhouyanhong@csu.edu.cn

E-mail:lgy@csu.edu.cn

Abbreviations: CD38, CD38 molecule; Akt, v-akt murine thymoma viral oncogene homolog 1; PI3K, phosphatidylinositol 3-kinase; MDM2, MDM2 oncogene, E3 ubiquitin protein ligase; TP53, tumor protein $\mathrm{p} 53$; GADPH, glyceraldehyde-3-phosphate dehydrogenase; IHC, immunohistochemistry

Key words: cervical cancer, CD38 molecule, expression, Akt, p53
The results of the present study suggested that CD38 is highly expressed in cervical carcinoma tissues and play an important role in dysregulation of the PI3K/Akt signaling pathway.

\section{Introduction}

Cervical cancer is the second most common cancer and the fifth most deadly malignancy in females worldwide, affecting 500,000 individuals each year. It is the leading cause of cancer mortality among women in developing countries (1). Invasion and metastasis are the major causes of cancer-related death. Persistent infection with high-risk types of human papillomavirus (HPV) is known to cause cervical cancer (2). However, additional genetic and epigenetic alterations are required for progression from precancerous disease to invasive cancer. Genomic imbalances can contribute to the deregulated expression of oncogenes and tumor-suppressor genes in cancer cells, and the accumulation of such altered genes has been correlated with tumor progression (3-5). DNA methylation is an early and frequent molecular alteration in cervical carcinogenesis. The dysregulated activation of genes, such as CD44 and SOX9 plays a role in cervical cancer (6-8). Genes amplified and suggested to be involved in cervical cancer, such as $S K P 2$, TERT, TRIO, RNASEN and PRKAAI, are overexpressed in tumor samples (9) and cell lines $(10,11)$. The inactivation of tumor-suppressor genes and the activation of oncogenes play a significant role in carcinogenesis. However, the etiology of cervical carcinoma remains poorly understood.

CD38 was originally defined as a T-cell activation/proliferation molecule (12). However, CD38 is currently defined as an ectoenzyme and a receptor $(13,14)$, while the expression of CD38 is not dependent on cell lineage or activation (15). Human CD38 is a multifunctional protein that triggers proliferation and differentiation. The enzyme shares extensive sequence similarity with Aplysia californica ADP-ribosyl cyclase (ADPRC). CD38 is known to be involved in activities typical of cell surface receptors, such as signaling for activation and proliferation events and heterotypic cell adhesion (16-18). 
CD38 contribution to disease progression and relapse in acute myeloid and chronic lymphocytic leukemia is well established and the expression of the enzyme is considered an important prognostic marker in leukemia (19-22).

The phosphatidylinositol 3-kinase (PI3K)/Akt pathway is known to play key roles in cell proliferation, apoptosis, cell survival in various cell types (23), and PIP3 is a lipid-signaling second messenger that further activates its downstream effectors such as Akt, inducing a conformational change in Akt that exposes the critical Thr308/309 residue to phosphorylation, and subsequently phosphorylated at Ser473/474 for full-length activation $(24,25)$. It has been previously demonstrated that PI3K/Akt signaling pathways regulate metastasis in a variety of cancer cells $(26,27)$.

In this study, we examined the expression levels of CD38 and the key molecular of PI3K/Akt signaling pathway in cervical cancer tissues. At the same time, the effects of CD38 were studied in vivo. Our results showed that CD38 was highly expressed in cervical carcinoma tissues and plays an important role in dysregulation of the PI3K/Akt signaling pathway.

\section{Materials and methods}

Cells culture. The Caski human cervical cancer cell line was cultured in RPMI-1640 supplemented with $10 \%$ fetal bovine serum (FBS) (both from Gibco by Life Technologies, Grand Island, NY, USA), $100 \mathrm{U} / \mathrm{ml}$ penicillin and $100 \mu \mathrm{g} / \mathrm{ml}$ streptomycin at $37^{\circ} \mathrm{C}$ in the presence of $5 \% \mathrm{CO}_{2}$.

Patient samples. Ten participants were recruited at the Third Xiangya Hospital, Central South University (Changsha, Hunan, China). Consent forms were obtained from individual patients, and experimental protocols were approved by the Institutional Review Board of the Third Xiangya Hospital. All 10 participants were female with histologically-confirmed cervical cancer (Table I). All the subjects enrolled in the study were Chinese. Cervical cancer and corresponding nontumor normal tissues were collected. Each biopsy sample was submitted to routine histological diagnosis, or quantitative polymerase chain reaction (qPCR), western blot analysis and immunohistochemistry (IHC).

Total RNA extraction and quantitative real-time polymerase chain reaction ( $R T-q P C R)$ analysis. Total RNA was extracted from the cervical cancer and corresponding non-tumor normal tissues with TRIzol reagent (Qiagen, Carlsbad, CA, USA). cDNA synthesis was carried out using the RevertAid First Strand cDNA Synthesis kit (CWBio, Beijing, China) according to the manufacturer's instructions. RT-qPCR was performed with GoTaq qPCR Master Mix (CWBio). The primers used for the RT-qPCR are shown in Table II. RT-qPCR was carried out with the Bio-Rad CFK96TM Real-Time system (Bio-Rad, Hercules, CA, USA). The data were analyzed by Bio-Rad CFK manager 2.0 software. The expression of mRNA was assessed by evaluating threshold cycle $(\mathrm{Ct})$ values and glyceraldehyde-3-phosphate dehydrogenase $(G A P D H)$ was used as an internal control GAPDH was calculated using the $2^{-\Delta \Delta \mathrm{Ct}}$ equation previously adopted by Livak et al (29): $\Delta \Delta \mathrm{Ct}=\left(\mathrm{Ct}_{\text {Target }}-\mathrm{Ct}_{\mathrm{GAPDH}}\right)$ cervical cancer - $\left(\mathrm{Ct}_{\mathrm{Target}}-\mathrm{Ct}_{\mathrm{GAPDH}}\right)$ control.
Table I. Characteristics of female cervical cancer patients diagnosed with squamous cell cancer.

\begin{tabular}{lccc}
\hline Samples & Age (years) & HPV type & Laborers $^{\mathrm{a}}$ \\
\hline A & 57 & $16,53,58$ & Yes \\
B & 49 & 16,58 & No \\
C & 53 & 18,35 & No \\
D & 51 & 16 & No \\
E & 45 & 33,58 & No \\
F & 41 & 16 & Yes \\
G & 50 & 16,58 & Yes \\
H & 43 & 16 & No \\
I & 48 & 16 & No \\
J & 62 & 52 & Yes \\
\hline
\end{tabular}

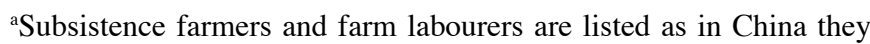
show a higher incidence of cervical cancer than other occupations. HPV, human papillomavirus.

Table II. List of human-specific primer sequences used in the study.

\begin{tabular}{lll}
\hline Target & \multicolumn{1}{c}{ Primer forward } & \multicolumn{1}{c}{ Primer reverse } \\
\hline CD20 & gggatctatgcacccatctg & tggagttttctccgttgct \\
CD21 & caaggcacaattccttggtt & tctaaggaactcccggtgtg \\
CD26 & caaattgaagcagccagaca & cacacttgaacacgccactt \\
CD34 & caagccaccagagctattcc & tccaccgttttccgtgtaat \\
CD38 & tgctgatgacctcacatggt & ccattgagcatcacatggac \\
CD73 & gccgctttagagaatgcaac & caggtttcgggaagatca \\
CD90 & cccagtgaagatgcaggttt & gacagcctgagagggtcttg \\
CD123 & ggacgtccagtacgacctgt & actttgagaaccgctggaga \\
CD133 & ttgtggcaaatcaccaggta & tcagatctgtgaacgccttg \\
\hline
\end{tabular}

To avoid false positive signals originating from DNA contamination, all human-specific PCR primers were designed with known amplicon size and where possible flanking a region that contains at least one intron.

IHC and evaluation of staining. IHC was performed using the peroxidase anti-peroxidase technique following a microwave antigen retrieval procedure. Antibody for $C D 38$ was purchased from Proteintech Biotechnology (Chicago, IL, USA). Antibody against $C D 38$ (1:100) was overlaid on cervical cancer and corresponding non-tumor normal tissue sections and incubated overnight at $4^{\circ} \mathrm{C}$. Secondary antibody incubation (Santa Cruz Biotechnology, Inc., Santa Cruz, CA, USA) was performed at room temperature for $30 \mathrm{~min}$.

The sections evaluated by two investigators in a blind manner in an effort to provide a consensus on staining patterns by light microscopy (Olympus, Tokyo, Japan). CD38 staining was assessed according to the methods described by Hara and Okayasu (28) with minor modifications. Each case was rated according to a score that added a scale of intensity of staining to the area of staining. At least 10 high-power fields were chosen 
randomly, and $>1,000$ cells were counted for each section. The intensity of staining was graded on the following scale: 0 , no staining; 1+, mild staining; $2+$, moderate staining; $3+$, intense staining. The area of staining was evaluated as follows: 0 , no staining of cells in any microscopic fields; $1+,<30 \%$ of tissue stained positive; $2+$, between 30 and $60 \%$ stained positive; $3+$, $>60 \%$ stained positive. The minimum score when summed (extension + intensity) was, therefore, 0 , and the maximum, 6. A combined staining score (extension + intensity) of $\leq 2$ was considered to be a negative staining (low staining), while a score between 3 and 4 was considered to be a moderate staining; whereas a score between 5 and 6 was considered to be a strong staining.

Expression analysis of miR-634, miR-664 and miR-140-5p in cervical cancer. Total RNA was extracted from the cervical cancer and corresponding non-tumor normal tissues with TRIzol reagent (Qiagen) according to the manufacturer's instructions. cDNA was synthesized from $2 \mathrm{mg}$ of total RNA with M-MLV Reverse Transcriptase (Promega, Fitchburg, WI, USA) in a $25 \mathrm{ml}$ volume $\{2 \mathrm{mg}$ total RNA, $400 \mathrm{mM}$ reverse transcription primer [oligo(dT) 18 for random primers for U6 rRNA and miR-634-, miR-664- and miR-140-5p-specific primers (Bulge-Loop ${ }^{\mathrm{TM}}$ miRNA qPCR primers from RiboBio, China) for miRNA], $4 \mathrm{U} / \mathrm{ml} \mathrm{M-MLV,} 1 \mathrm{U} / \mathrm{ml}$ inhibitor, $0.4 \mathrm{mM}$ dNTP mix\}. qPCR was carried out with the reagents of a SYBR-Green I mix (Takara, Dalian, China) in a $20 \mathrm{ml}$ reaction volume (10 $\mathrm{ml}$ SYBR-Green I mix, $200 \mathrm{mM}$ forward and reverse primer, $2 \mathrm{ml}$ cDNA template) on an MJ Opticon Monitor Chromo4 instrument (Bio-Rad) using the following protocol: $95^{\circ} \mathrm{C}$ for $20 \mathrm{sec} ; 40$ cycles of $95^{\circ} \mathrm{C}$ for $10 \mathrm{sec}, 60^{\circ} \mathrm{C}$ for $20 \mathrm{sec}$ and $70^{\circ} \mathrm{C}$ for $1 \mathrm{sec}$. Data analysis was performed using the $2^{-\Delta \Delta C t}$ method $(6,29)$.

Construction of pEGFP-N1-CD38 vector. The coding region of CD38 gene was generated by PCR with the primer pair 5'-ATACTCGAGATGGCCAACTGCGAGTTCAG-3' and 5'-GCGAAGCTTTCAGATCTCAGATGTGCAAG-3'. The PCR was performed under the following conditions: one cycle for $5 \mathrm{~min}$ at $94^{\circ} \mathrm{C} ; 30$ cycles for $45 \mathrm{sec}$ at $94^{\circ} \mathrm{C}, 45 \mathrm{sec}$ at $55^{\circ} \mathrm{C}$, and $90 \mathrm{sec}$ at $72^{\circ} \mathrm{C}$, and ended with $10 \mathrm{~min}$ at $72^{\circ} \mathrm{C}$. The fragments were cloned into the TA vector (Promega) and used to transform E. coli JM109 (Takara). Following selection and propagation, the pure plasmid DNA was prepared by standard methods. The DNA fragments were removed from the TA vector by restriction enzyme digestion with XhoI and HindIII (Promega) to subclone into the pEGFP-N1 vector. The fusion sequences were verified by DNA sequencing using ABI 3730.

Cell transfection. Cell transfection was achieved by using Lipofectamine, according to the manufacturer's instructions (Life Technologies, Grand Island, NY, USA). Cells $\left(2 \times 10^{5}\right)$ were placed in each well of a 6 -well plate $24 \mathrm{~h}$ prior to the transfection. For each transfection, $2 \mu \mathrm{g}$ of pEGFP-N1-CD38 plasmid and pEGFP-N1 vector plasmid was transfected into Caski cells, respectively. The plasmids were diluted with $100 \mu \mathrm{l}$ of serum-free media and $4 \mu \mathrm{l}$ Lipofectamine was added into $100 \mu 1$ serum-free media. The two solutions were combined, mixed gently and incubated at room temperature for $30 \mathrm{~min}$.
The $200 \mu 1$ mixture and $200 \mu 1$ of serum-free media were added into each well. The cells were then incubated at $37^{\circ} \mathrm{C}$ for $24 \mathrm{~h}$, followed by replacing the transfection media with fresh complete culture media. After an additional $48 \mathrm{~h}$ culture, the cells were harvested for the western blot analysis.

Western blot analysis. The cervical cancer and corresponding non-tumor normal tissues, and Caski cells were lysed in RIPA buffer (CWBio) and total protein concentration was determined using Pierce ${ }^{\circledR}$ BCA Protein assay kit (Thermo Scientific, Inc., Rockford, IL, USA). Extracts containing $50 \mu \mathrm{g}$ of proteins were separated in 10\% SDS-PAGE gels and electroblotted onto nitrocellulose membranes (HyClone Laboratories, Logan, UT, USA). The membranes were inhibited using Tris-buffered saline/Tween-20 (25 mM Tris- $\mathrm{HCl}$, $150 \mathrm{mM} \mathrm{NaCl}, \mathrm{pH} 7.5$ and $0.05 \%$ Tween-20) containing 5\% non-fat milk followed by overnight incubation at $4{ }^{\circ} \mathrm{C}$ with primary antibodies [rabbit anti-PI3K antibody, 1:500; and rabbit anti-Akt antibody, 1:300 (Cell Signalling Technology, USA); rabbit anti-MDM2 antibody, 1:200 and rabbit anti-p53 antibody, 1:200 (Wuhan Boster, Wuhan, China)]. Following three washes, the membranes were incubated with horseradish peroxidase-conjugated secondary antibodies (Santa Cruz Biotechnology, Inc.) and the specific signals were visualized using an ECL detection system. Anti-GAPDH antibody (1:3,000; Santa Cruz Biotechnology, Inc.) was used as a loading control.

Statistical analysis. Differences of non-parametric variables were analyzed by the Mann-Whitney U test. Differences of the quantitative variables between groups were analyzed by the Student's t-test using SPSS 11.0 program (SPSS, Chicago, IL, USA). $\mathrm{P}<0.05$ was considered statistically significant.

\section{Results}

Detection of mRNA expression levels of the CD molecules in cervical cancer. To detect the mRNA expression levels of the CD molecules in cervical cancer and the adjacent non-cancerous tissues, 10 samples of each were selected to perform qPCR of the $C D 20, C D 21, C D 26, C D 34, C D 38$, $C D 73, C D 90, C D 123$ and $C D 133$ genes. Data were analyzed using the $2^{-\Delta \Delta \mathrm{Ct}}$ method. The fold-change in the expression of these genes relative to the internal control gene, $G A P D H$, was also analyzed. The expression of the $C D 38, C D 34$ and $C D 90$ genes was higher in the cervical cancer samples compared with the adjacent non-cancerous tissues and the normalized $C D 38, C D 34$ and $C D 90$ gene expression in the cervical cancer samples was upregulated by 4.40-, 2.71- and 2.64-fold, respectively (Table III). The expression levels were not significantly different for CD20, CD26, CD73 and CD133 between the cervical cancer and adjacent non-cancerous tissues (Table III). Thus, we selected to determine the funciton of CD38 and its underlying mechanism in cervical cancer.

Western blot analysis of protein expression levels of CD38 in cervical cancer. To determine whether the $C D 38$ gene was expressed at a higher level in the cervical cancer compared with the adjacent non-cancerous tissues, the protein expression levels of CD38 were further examined by western blot analysis 
Table III. Identification of the mRNA expression level of the CD molecules in cervical cancer and adjacent non-cancerous tissues by qPCR.

\begin{tabular}{llllcccc}
\hline Gene & \multicolumn{1}{c}{ Sample } & $\mathrm{n}$ & $\begin{array}{c}\text { Gene Ct } \\
(\text { mean } \pm \mathrm{SD})\end{array}$ & $\begin{array}{c}\text { GAPDH Ct } \\
(\text { mean } \pm \mathrm{SD})\end{array}$ & $\begin{array}{c}\Delta \mathrm{Ct} \\
(\text { mean } \pm \mathrm{SD})\end{array}$ & $\begin{array}{c}\Delta \Delta \mathrm{Ct} \\
(\text { mean } \pm \mathrm{SD})\end{array}$ & Fold $^{\mathrm{a}}$ \\
\hline CD20 & Cervical cancer & 10 & $31.56 \pm 1.46$ & $19.43 \pm 0.76$ & $12.13 \pm 1.17$ & $0.07 \pm 0.04$ & 0.95 \\
& Non-cancerous tissues & 10 & $33.97 \pm 0.71$ & $21.91 \pm 0.94$ & $12.06 \pm 0.85$ & & $(0.93-0.98)$ \\
CD21 & Cervical cancer & 10 & $29.07 \pm 1.69$ & $19.43 \pm 0.76$ & $9.64 \pm 1.12$ & $-0.31 \pm 0.13$ & 1.23 \\
& Non-cancerous tissues & 10 & $31.86 \pm 0.61$ & $21.91 \pm 0.94$ & $9.95 \pm 0.74$ & & $(1.13-1.35)$ \\
CD26 & Cervical cancer & 10 & $31.69 \pm 1.73$ & $19.43 \pm 0.76$ & $12.26 \pm 1.04$ & $-0.10 \pm 0.06$ & 1.07 \\
& Non-cancerous tissues & 10 & $34.27 \pm 0.78$ & $21.91 \pm 0.94$ & $12.36 \pm 0.81$ & & $(1.02-1.12)$ \\
CD34 & Cervical cancer & 10 & $33.22 \pm 1.92$ & $19.43 \pm 0.76$ & $13.79 \pm 1.28$ & $-1.44 \pm 0.47$ & 2.71 \\
& Non-cancerous tissues & 10 & $37.14 \pm 1.12$ & $21.91 \pm 0.94$ & $15.23 \pm 1.04$ & & $(1.96-3.76)$ \\
CD38 & Cervical cancer & 10 & $30.42 \pm 1.77$ & $19.43 \pm 0.76$ & $10.99 \pm 1.37$ & $-2.14 \pm 0.85$ & 4.40 \\
& Non-cancerous tissues & 10 & $35.04 \pm 1.42$ & $21.91 \pm 0.94$ & $13.13 \pm 1.22$ & & $(2.45-7.94)$ \\
CD73 & Cervical cancer & 10 & $31.69 \pm 1.73$ & $19.43 \pm 0.76$ & $12.26 \pm 1.39$ & $-0.45 \pm 0.19$ & 1.37 \\
& Non-cancerous tissues & 10 & $34.62 \pm 0.78$ & $21.91 \pm 0.94$ & $12.71 \pm 0.84$ & & $(1.20-1.57)$ \\
CD90 & Cervical cancer & 10 & $30.93 \pm 0.98$ & $19.43 \pm 0.76$ & $11.50 \pm 0.86$ & $-1.40 \pm 0.42$ & 2.64 \\
& Non-cancerous tissues & 10 & $34.81 \pm 1.05$ & $21.91 \pm 0.94$ & $12.90 \pm 0.97$ & & $(1.97-3.53)$ \\
CD123 & Cervical cancer & 10 & $31.70 \pm 1.79$ & $19.43 \pm 0.76$ & $12.27 \pm 1.34$ & $-0.84 \pm 0.38$ & 1.79 \\
& Non-cancerous tissues & 10 & $35.02 \pm 0.42$ & $21.91 \pm 0.94$ & $13.11 \pm 0.59$ & & $(1.37-2.31)$ \\
CD133 & Cervical cancer & 10 & $32.61 \pm 2.91$ & $19.43 \pm 0.76$ & $13.18 \pm 1.90$ & $-0.04 \pm 0.02$ & 1.02 \\
& Non-cancerous tissues & 10 & $35.13 \pm 1.85$ & $21.91 \pm 0.94$ & $13.22 \pm 1.45$ & & $(1.01-1.04)$ \\
\hline
\end{tabular}

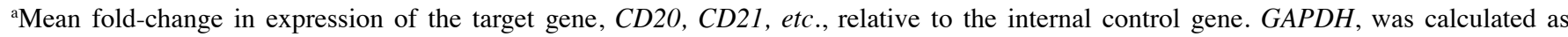
described in 'Materials and methods'. At least three replicates of each reaction were performed. Ct, threshold cycle; qPCR, quantitative polymerase chain reaction; GAPDH, glyceraldehyde-3-phosphate dehydrogenase; SD, standard deviation.

Table IV. The difference of CD38 expression between cervical cancer and the adjacent non-cancerous tissues.

\begin{tabular}{lccccc}
\hline & & \multicolumn{3}{c}{ Score } \\
\cline { 3 - 6 } Tissues & $\mathrm{n}$ & $\begin{array}{c}\text { Low (0-2) } \\
\mathrm{n}(\%)\end{array}$ & $\begin{array}{c}\text { Moderate }(3-4) \\
\mathrm{n}(\%)\end{array}$ & $\begin{array}{c}\text { High (5-6) } \\
\mathrm{n}(\%)\end{array}$ & P-value \\
\hline Cervical cancer & 30 & $7(23.3)$ & $11(36.7)$ & $12(40.0)$ & $=0.02<0.05$ \\
Non-cancerous & 30 & $17(56.7)$ & $8(26.7)$ & $5(16.6)$ & \\
\hline
\end{tabular}

$\mathrm{P}<0.05$ by Mann-Whitney U test.

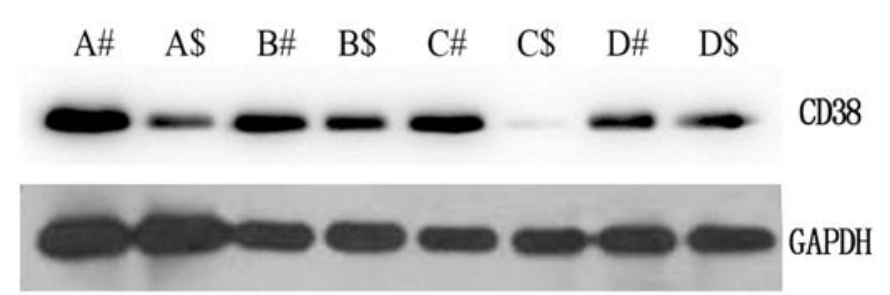

Figure 1. Expression levels of the CD38 protein in cervical cancer and the adjacent non-cancerous tissues. In total, A, B, C and D tissues which were used in the detection of mRNA expression levels by qPCR were selected to detect the expression levels of CD38 protein by western blot analysis. \#, denotes cervical cancer and $\$$, denotes the adjacent non-cancerous tissues. Data are representative of three independent experiments.
(Fig. 1). In comparison with the adjacent non-cancerous tissues, the expression level was identified to be greater in cervical cancer tissues, which corresponded with the qPCR results. These results suggested that CD38 is highly expressed in cervical cancer.

IHC analysis of protein expression levels of CD38 in cervical cancer. IHC was carried out with antibodies against CD38 protein in the cervical cancer and adjacent non-cancerous tissues. CD38 was identified as differentially expressed between the cervical cancer and adjacent non-cancerous tissues. IHC showed a similar pattern in protein expression, which was similar to that of the western blot results. There was 

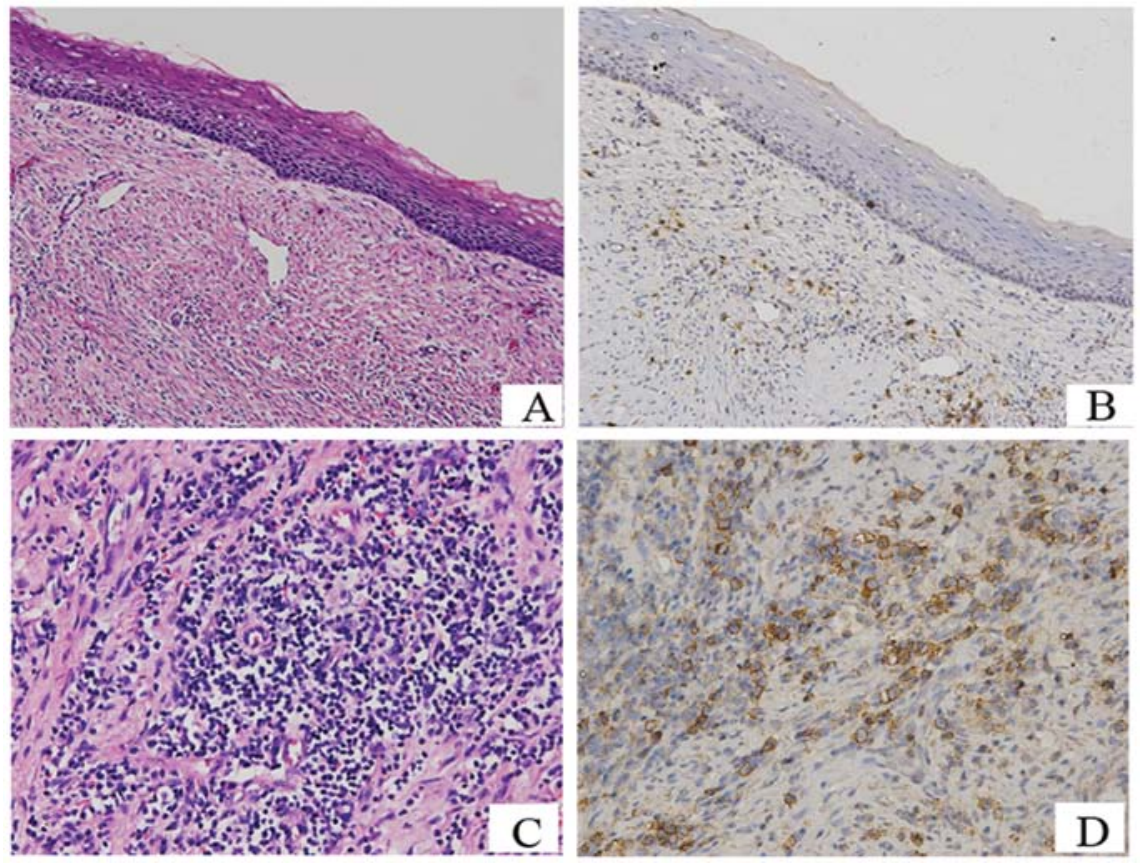

Figure 2. Immunohistochemical analysis of the expression of CD38 protein in the cervical cancer and adjacent non-cancerous tissues. Antibody of CD38 protein was used; brown grains denote a positive signal. H\&E staining of (A) cervical epithelial tissue, (B) CD38 staining of cervical epithelial tissue, (C) H\&E staining of cervical cancer tissue, (D) CD38 staining of cervical cancer tissue. Original magnification, x200.

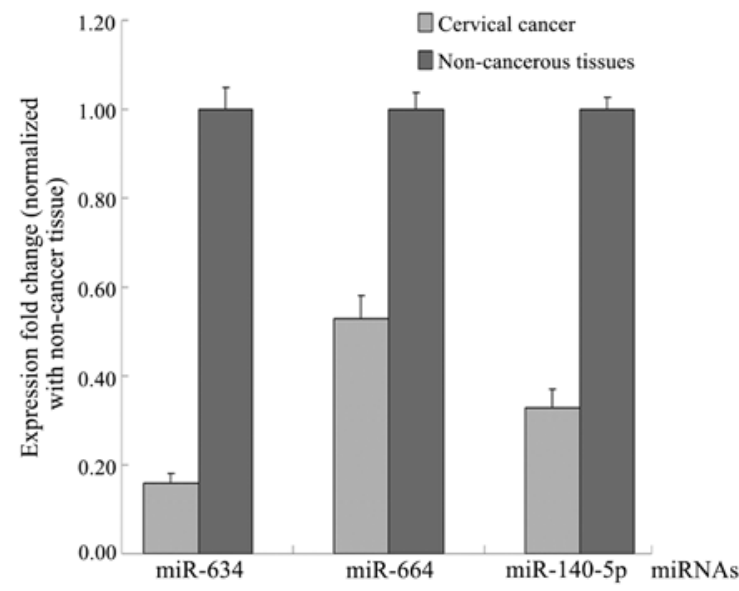

Figure 3. The expression level of miR-634, miR-664 and miR-140-5p between the cervical cancer and adjacent non-cancerous tissues.

a $40 \%(12 / 30)$ high score of CD38 in the cervical cancer and $16.6 \%(5 / 30)$ in the adjacent non-cancerous tissues. The distribution of the low score was $23.3 \%$ (7/30) and $56.7 \%(17 / 30)$ in the cervical cancer and adjacent non-cancerous tissues, respectively $(\mathrm{P}=0.02<0.05)$ (Fig. 1) (Table IV). The IHC results corresponded with those of the qPCR results.

miR-634, miR-664 and miR-140-5p which regulate CD38 exhibit a low expression in cervical cancer tissues. As CD38 is a potential miR target, the open access programs, TargetScan (http://www.targetscan.org/), PicTar (http://pictar.mdc-berlin. $\mathrm{de} /$ ) and miRBase (http://mirbase.org/index.shtml), were used to predict the targets of miR-634, miR-664 and miR140-5p. The endogenous expression of miR-634, miR-664 and miR-140-5p was compared between the cervical cancer and

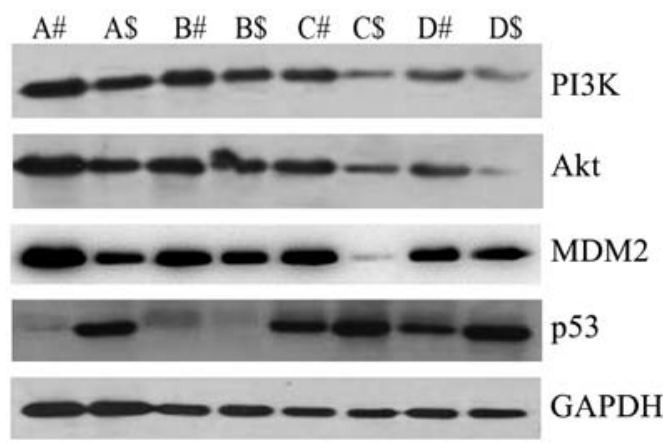

Figure 4. Expression levels of phosphatidylinositol 3-kinase (PI3K), Akt, MDM2, and p53 protein in cervical cancer and the adjacent non-cancerous tissues. In total, A, B, C and D tissues which were used in the detection of mRNA expression levels by qPCR were selected to detect the expression levels of PI3K, Akt, MDM2 and p53 protein by western blot analysis. \#, denotes cervical cancer and $\$$, denotes the adjacent non-cancerous tissues. Data are representative of three independent experiments.

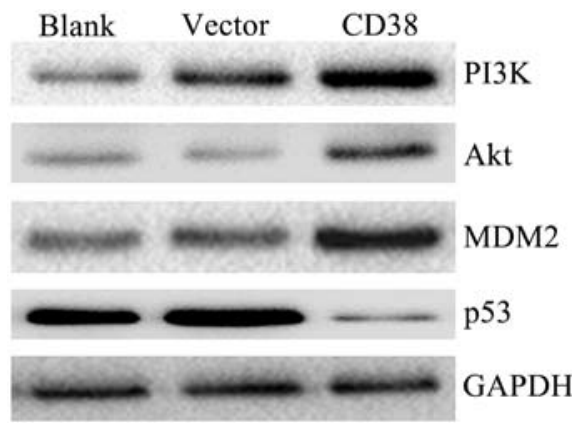

Figure 5. Expression levels of phosphatidylinositol 3-kinase (PI3K), Akt, MDM2 and p53 protein in the Caski cervical cancer cell line. CD38 denotes Caski cells transfected with pEGFP-N1-CD38. Vector denotes Caski cells transfected with pEGFP-N1. Blank denotes Caski cells that have not been transfected with plasmid. Data are representative of three independent experiments. 
adjacent non-cancerous tissues by qPCR. The expression of miR-634, miR-664 and miR-140-5p was downregulated in the cervical cancer tissues (Fig. 3). These results suggested that CD38 was upregulated in the cervical cancer as compared with the non-cancerous tissues.

CD38 is correlated with dysregulation of the PI3K/Akt signaling pathway in cervical cancer tissues in vitro. To determine the possible mechanism of CD38 in cervical cancer, we examined the expression levels of key molecules in the PI3K/Akt signaling pathway by western blot analysis. PI3K and Akt were upregulated in cervical cancer compared with the adjacent non-cancerous tissues. MDM2 had the same tendency as PI3K and Akt. However, p53 was downregulated in cervical cancer (Fig. 4). Combined with the above result showing CD38 was highly expressed in cervical cancer, the results suggested that $\mathrm{CD} 38$ is correlated with dysregulation of the PI3K/Akt signaling pathway in cervical cancer tissues in vitro.

CD38 overexpression affects the expression of PI3K, Akt, $M D M 2$ and $p 53$ in vivo. To confirm whether CD38 affects the expression of PI3K, Akt, MDM2 and p53 in vivo, we constructed the plasmid of pEGFP-N1-CD38. The plasmid of pEGFP-N1-CD38 and pEGFP-N1 was transfected into the Caski cervical cancer cell line. The cells were collected after $48 \mathrm{~h}$ with transfection and the expression levels of PI3K, Akt, MDM2 and 553 proteins were examined in vivo. The PI3K, Akt and MDM2 were upregulated in Caski cells in which CD38 was overexpressed. At the same time, p53 was downregulated in Caski cells in which CD38 was overexpressed (Fig. 5). The results suggested that CD38 overexpression affected the expression of PI3K, Akt, MDM2 and $\mathrm{p} 53$ in vivo.

\section{Discussion}

Approximately 530,000 new cervical cancer cases are diagnosed annually worldwide, with 275,000 individuals succumbing from the disease (30). The disease is largely preventable. Persistent infection with high-risk types of HPV is known to cause cervical cancer. However, the inactivation of tumor-suppressor genes and activation of oncogenes play a significant role in the progression from precancerous disease to invasive cancer, caused by the genetic and epigenetic alterations (31-34). The etiology of cervical carcinoma remains poorly understood.

Human CD38 is a multifunctional protein that triggers proliferation and differentiation, contributing to disease progression and relapse in certain types of cancer (20-22). To the best of our knowledge, no studies have been conducted on the relationship between CD38 and cervical cancer. In this study, we detected the mRNA expression levels of CD38 in cervical cancer and found that CD38 was upregulated by 4.40 -fold in cervical cancer compared with the adjacent non-cancerous tissues. The protein expression level of CD38 exhibited the same tendency as the mRNA level in cervical cancer as indicated by the western blot analysis results. At the same time, the results of IHC showed that there was a $40 \%(12 / 30)$ high score of CD38 in cervical cancer tissues and $16.6 \%(5 / 30)$ in the adjacent non-cancerous tissues $(\mathrm{p}=0.02<0.05)$. Perenkov et al (35) found that the expression of the $C D 38$ gene is heterogeneous in the tumor cells of patients with colorectal cancer. CD38 expression is triggered at least in part by a certain cytokine(s) secreted by cancer cells (36). Our data are consistent with those observations and suggest that CD38 maybe play an important role in cervical cancer.

To verify CD38 is highly expressed in cervical cancer, we detected the expression of miR-634, miR-664 and miR-140-5p, which were the predicted target genes of CD38. Our data show that the expression of miR-634, miR-664 and miR-140-5p were downregulated in the cervical cancer tissues. Yang et al (37) found that the upregulation of miR-664, miR-485-3p and miR-495 contributed to a lower MAT1A expression in hepatocellular carcinoma (HCC), and enhanced tumorigenesis may provide potential targets for HCC therapy. MicroRNA-140-5p was significantly decreased in HCC tissues and its expression levels were correlated with multiple nodules, vein invasion, capsular formation, and differentiation of HCC (38). Our results demonstrate that the expression level of CD38 was high in cervical cancer as compared with that of the miRNA level.

To explore the possible mechanism of CD38 in cervical cancer, we detected the expression levels of key molecules in the $\mathrm{PI} 3 \mathrm{~K} / \mathrm{Akt}$ signaling pathway by western blot analysis in vitro and found that PI3K, Akt and MDM2 were upregulated in cervical cancer, but p53 was downregulated in cervical cancer. Furthermore, we studied whether CD38 affected the expression of PI3K, Akt, MDM2 and $\mathrm{p} 53$ in vivo. CD38 overexpression is able to increase the expression of PI3K, Akt and MDM2, and suppress the expression of p53 in vivo. It has been demonstrated that PI3K/Akt signaling pathways regulate metastasis in a variety of cancer cells $(23,26,27)$. Through the regulation of the expression of downstream target genes, p53 regulates cell cycle arrest, apoptosis, senescence, cellular energy metabolism and anti-oxidant defense.

In conclusion, our results have shown that CD38 was highly expressed in cervical carcinoma tissues and play an important role in the dysregulation of the PI3K/Akt signaling pathway.

\section{Acknowledgements}

This study was supported by the National Natural Science Foundation of China (81272975, 81402270), the Key Project of Hunan Provincial Natural Science Foundation (12JJ2044), the Project of Hunan Provincial Natural Science Foundation (12JJ3121), the Project of Hunan Provincial Development and Reform Commission, the Planned Science and Technology Project of Hunan Province (2010FJ3088 and 2012FJ2014), and the Key Project of Hunan Provincial Natural Science Foundation (12JJ2044).

\section{References}

1. Jemal A, Bray F, Center MM, Ferlay J, Ward E and Forman D: Global cancer statistics. CA Cancer J Clin 61: 69-90, 2011.

2. Yugawa T and Kiyono T: Molecular mechanisms of cervical carcinogenesis by high-risk human papillomaviruses: novel functions of E6 and E7 oncoproteins. Rev Med Virol 19: 97-113, 2009.

3. Bozic I, Antal T, Ohtsuki H, Carter H, Kim D, Chen S, Karchin R, Kinzler KW, Vogelstein B and Nowak MA: Accumulation of driver and passenger mutations during tumor progression. Proc Natl Acad Sci USA 107: 18545-18550, 2010. 
4. Kirchhoff M, Rose H, Petersen BL, Maahr J, Gerdes T, Lundsteen C, Bryndorf T, Kryger-Baggesen N, Christensen L, Engelholm SA and Philip J: Comparative genomic hybridization reveals a recurrent pattern of chromosomal aberrations in severe dysplasia/carcinoma in situ of the cervix and in advanced-stage cervical carcinoma. Genes Chromosomes Cancer 24: 144-150, 1999.

5. Heselmeyer K, Macville M, Schröck E, Blegen H, Hellström AC, Shah K, Auer G and Ried T: Advanced-stage cervical carcinomas are defined by a recurrent pattern of chromosomal aberrations revealing high genetic instability and a consistent gain of chromosome arm 3q. Genes Chromosomes Cancer 19: 233-240, 1997.

6. Xiao S, Zhou Y, Jiang J, Yuan L and Xue M: CD44 affects the expression level of FOS-like antigen 1 in cervical cancer tissues. Mol Med Rep 9: 1667-1674, 2014.

7. Ibrahim EM, Stewart RL, Corke K, Blackett AD, Tidy JA and Wells M: Upregulation of CD44 expression by interleukins 1,4 , and 13 , transforming growth factor-beta1, estrogen, and progestogen in human cervical adenocarcinoma cell lines. Int $\mathrm{J}$ Gynecol Cancer 16: 1631-1642, 2006.

8. Wobus M, Kuns R, Wolf C, Horn LC, Köhler U, Sheyn I, Werness BA and Sherman LS: CD44 mediates constitutive type I receptor signaling in cervical carcinoma cells. Gynecol Oncol 83: $227-234,2001$

9. Scotto L, Narayan G, Nandula SV, Subramaniyam S, Kaufmann AM, Wright JD, Pothuri B, Mansukhani M, Schneider A, Arias-Pulido H and Murty VV: Integrative genomics analysis of chromosome 5p gain in cervical cancer reveals target over-expressed genes, including Drosha. Mol Cancer 7: 58, 2008.

10. Dowen SE, Neutze DM, Pett MR, Cottage A, Stern P, Coleman N and Stanley MA: Amplification of chromosome 5p correlates with increased expression of Skp2 in HPV-immortalized keratinocytes. Oncogene 22: 2531-2540, 2003.

11. Kloth JN, Oosting J, van Wezel T, Szuhai K, Knijnenburg J, Gorter A, Kenter GG, Fleuren GJ and Jordanova ES: Combined array-comparative genomic hybridization and single-nucleotide polymorphism-loss of heterozygosity analysis reveals complex genetic alterations in cervical cancer. BMC Genomics 8: 53 , 2007.

12. Funaro A, Spagnoli GC, Ausiello CM, Alessio M, Roggero S, Delia D, Zaccolo $\mathrm{M}$ and Malavasi F: Involvement of the multilineage CD38 molecule in a unique pathway of cell activation and proliferation. J Immunol 145: 2390-2396, 1990.

13. Howard M, Grimaldi JC, Bazan JF, Lund FE, Santos-ArgumedoL, Parkhouse RM, Walseth TF and Lee HC: Formation and hydrolysis of cyclic ADP-ribose catalyzed by lymphocyte antigen CD38. Science 262: 1056-1059, 1993.

14. Malavasi F, Funaro A, Roggero S, Horenstein A, Calosso L and Mehta K: Human CD38: a glycoprotein in search of a function. Immunol Today 15: 95-97, 1994.

15. Malavasi F, Deaglio S, Funaro A, Ferrero E, Horenstein AL, Ortolan E, Vaisitti T and Aydin S: Evolution and function of the ADP ribosyl cyclase/CD38 gene family in physiology and pathology. Physiol Rev 88: 841-886, 2008.

16. Karimi-Busheri F, Zadorozhny V, Shawler DL and Fakhrai H: The stability of breast cancer progenitor cells during cryopreservation: maintenance of proliferation, self-renewal, and senescence characteristics. Cryobiology 60: 308-314, 2010

17. Karimi-Busheri F, Zadorozhny V, Li T, Lin H, Shawler DL and Fakhrai H: Pivotal role of CD38 biomarker in combination with CD24, EpCAM, and ALDH for identification of H460 derived lung cancer stem cells. J Stem Cells 6: 9-20, 2011.

18. Karimi-Busheri F, Zadorozhny V, Carrier E and Fakhrai $\mathrm{H}$ Molecular integrity and global gene expression of breast and lung cancer stem cells under long-term storage and recovery. Cell Tissue Bank 14: 175-186, 2013.

19. Malavasi F, Deaglio S, Damle R, Cutrona G, Ferrarini M and Chiorazzi N: CD38 and chronic lymphocytic leukemia: a decade later. Blood 118: 3470-3478, 2011.
20. Hamblin TJ: CD38: what is it there for? Blood 102: 1939-1940, 2003.

21. Jawad M, Yu N, Seedhouse CH, Tandon K, Russell NH and Pallis M: Targeting of CD34 ${ }^{+} \mathrm{CD} 38^{-}$cells using Gemtuzumab ozogamicin (Mylotarg) in combination with tipifarnib (Zarnestra) in acute myeloid leukaemia. BMC Cancer 12: 431, 2012

22. Dürig J, Naschar M, Schmücker U, Renzing-Köhler K, Hölter T, Hüttmann A and Dührsen U: CD38 expression is an important prognostic marker in chronic lymphocytic leukaemia. Leukemia 16: 30-35, 2002.

23. Qiao M, Sheng S and Pardee AB: Metastasis and Akt activation. Cell Cycle 7: 2991-2996, 2008.

24. Kreisberg JI, Malik SN, Prihoda TJ, Bedolla RG, Troyer DA, Kreisberg S and Ghosh PM: Phosphorylation of Akt (Ser473) is an excellent predictor of poor clinical outcome in prostate cancer. Cancer Res 64: 5232-5236, 2004.

25. Malik SN, Brattain M, Ghosh PM, Troyer DA, Prihoda T, Bedolla R and Kreisberg JI: Immunohistochemical demonstration of phospho-Akt in high Gleason grade prostate cancer. Clin Cancer Res 8: 1168-1171, 2002

26. Vivanco I and Sawyers CL: The phosphatidylinositol 3-kinase Akt pathway in human cancer. Nat Rev Cancer 2: 489-501, 2002.

27. Wagner EF and Nebreda AR: Signal integration by JNK and p38 MAPK pathways in cancer development. Nat Rev Cancer 9: 537-549, 2009.

28. Hara A and Okayasu I: Cyclooxygenase-2 and inducible nitric oxide synthase expression in human astrocytic gliomas: correlation with angiogenesis and prognostic significance. Acta Neuropathol 108: 43-48, 2004.

29. Livak KJ and Schmittgen TD: Analysis of relative gene expression data using real-time quantitative PCR and the 2(-Delta Delta C(T)) method. Methods 25: 402.408, 2001.

30. IARC Globocan: Cervical Cancer Incidence and Mortality Worldwide in 2008. http://globocan.iarc.fr/factsheets/cancers/ cervix.asp.

31. Alameda F, Espinet B, Corzo C, Muñoz R, Bellosillo B Lloveras B, Pijuan L, Gimeno J, Salido M, Solé F, Carreras R and Serrano S: 3q26 (hTERC) gain studied by fluorescence in situ hybridization as a persistence-progression indicator in low-grade squamous intraepithelial lesion cases. Hum Pathol 40: 1474-1488, 2009.

32. Ma YY, Wei SJ, Lin YC, Lung JC, Chang TC, Whang-Peng J, Liu JM, Yang DM, Yang WK and Shen CY: PIK3CA as an oncogene in cervical cancer. Oncogene 19: 2739-2744, 2000.

33. Vazquez-Mena $\mathrm{O}$, Medina-Martinez I, Juárez-Torres E, Barrón V, Espinosa A, Villegas-Sepulveda N, Gómez-Laguna L, Nieto-Martínez K, Orozco L, Roman-Basaure E, Muñoz Cortez S, Borges Ibañez M, Venegas-Vega C, Guardado-Estrada M, Rangel-López A, Kofman S and Berumen J: Amplified genes may be overexpressed, unchanged, or downregulated in cervical cancer cell lines. PLoS One 7: e32667, 2012.

34. Andersson S, Wallin KL, Hellström AC, Morrison LE, Hjerpe A, Auer G, Ried T, Larsson C and Heselmeyer-Haddad K: Frequent gain of the human telomerase gene TERC at $3 q 26$ in cervical adenocarcinomas. Br J Cancer 95: 331-338, 2006.

35. Perenkov AD, Novikov DV, Sakharnov NA, Aliasova AV, Utkin OV, Baryshnikov AIu and Novikov VV: Heterogeneous expression of CD38 gene in tumor tissue in patients with colorectal cancer. Mol Biol (Mosk) 46: 786-791, 2012 (In Russian).

36. Albeniz I, Demir-Coşkun O, Türker-Şener L, Bas A, Asoğlu O and Nurten R: CD38 expression as response of hematopoietic system to cancer. Oncol Lett 2: 659-664, 2011.

37. Yang H, Cho ME, Li TW, Peng H, Ko KS, Mato JM and Lu SC: MicroRNAs regulate methionine adenosyltransferase $1 \mathrm{~A}$ expression in hepatocellular carcinoma. J Clin Invest 123: 285-298, 2013.

38. Yang H, Fang F, Chang R and Yang L: MicroRNA-140-5p suppresses tumor growth and metastasis by targeting transforming growth factor $\beta$ receptor 1 and fibroblast growth factor 9 in hepatocellular carcinoma. Hepatology 58: 205-217, 2013. 